\title{
Polysèmes
}

Revue d'études intertextuelles et intermédiales

\section{Éthique et esthétique néoréalistes dans In Custody d'Anita Desai : les valeurs du noir et blanc contre l'exotisme de la couleur}

Neorealist Ethics and Aesthetics in Anita Desai's In Custody: The Values of Black and White versus the Exoticism of Colours

\section{Anne Le Guellec-Minel}

\section{OpenEdition}

Journals

Édition électronique

URL : http://journals.openedition.org/polysemes/542

DOI : $10.4000 /$ polysemes. 542

ISSN : 2496-4212

Éditeur

SAIT

\section{Référence électronique}

Anne Le Guellec-Minel, «Éthique et esthétique néoréalistes dans In Custody d'Anita Desai : les valeurs du noir et blanc contre l'exotisme de la couleur », Polysèmes [En ligne], 14 | 2015, mis en ligne le 18 novembre 2015, consulté le 10 octobre 2020. URL : http://journals.openedition.org/polysemes/542 ; DOI : https://doi.org/10.4000/polysemes.542

Ce document a été généré automatiquement le 10 octobre 2020

Polysèmes 


\title{
Éthique et esthétique néoréalistes dans In Custody d'Anita Desai : les valeurs du noir et blanc contre l'exotisme de la couleur
}

\author{
Neorealist Ethics and Aesthetics in Anita Desai's In Custody: The Values of \\ Black and White versus the Exoticism of Colours
}

Anne Le Guellec-Minel

\section{NOTE DE L'AUTEUR}

Une version différente de cet article, intitulée « Strategies of Colour, Mysticism of Form in Anita Desai's In Custody » a été publiée dans la revue Commonwealth Essays and Studies 32.2 (Spring 2010):75-86.

1 En 1984, Anita Desai publiait In Custody (ci-après IC), qui relate les péripéties tragicomiques accompagnant les efforts vains de Deven Sharma, universitaire médiocre et poète raté, pour faire retrouver à un vieux poète ourdouphone sa grandeur passée, dans le contexte peu propice d'une Inde post-Partition fascinée par le modèle de prospérité américain et japonais. Le roman marquait un tournant dans la carrière de Desai, dans la mesure où, contrairement aux romans précédents, il était centré sur un personnage masculin. Ce changement répondait au besoin de l'auteur « d'ouvrir les portes, d'élargir la toile » (Costa), et fut salué par la critique comme une évolution positive vers le réalisme. En 1993, Ismail Merchant, qui était lui-même d'origine indienne ourdouphone et, de ce fait, sensibilisé au déclin de l'aura culturelle de l'ourdou littéraire en Inde, adaptait le roman pour son premier long-métrage en tant que réalisateur, d'après un script d'Anita Desai. Le film obtint plusieurs prix du gouvernement indien. Cependant Desai a dit avoir été stupéfaite, voire outrée ( « shocked »), en visionnant l'adaptation qui avait été faite de son univers romanesque : 
« In my imagination it was all very grey, very dark and dirty, and I just couldn't believe my eyes when I saw it in gorgeous Technicolor, and everyone beautifully dressed, looking very handsome... That's the world of Ismail Merchant; he makes everything look so beautiful, gorgeous. [...] It's his vision of the book. He is very happy with it. It is not my vision: I would have preferred it in black and white, more in the school of New Realism » (Costa).

2 C'est ce rejet du Technicolor par Anita Desai, et sa référence au noir et blanc du Nouveau Réalisme comme métaphore visuelle de sa technique romanesque, qui ont servi de point de départ à notre réflexion qui s'intéressera au traitement de la couleur dans une problématique relevant tant de l'éthique que de l'esthétique. Il s'agira notamment de tenter de situer la grisaille dominante du roman entre un vérisme engagé et un symbolisme moderniste plus abstrait, mais aussi de relever et d'interpréter les occurrences de la couleur résiduelle, particulièrement dans sa dimension agressive ou repoussante, à contre-courant de tout exotisme vitaliste. On s'interrogera enfin sur la possibilité de lire la référence encore plus discrète au noir et blanc de la calligraphie et à l'abstraction géométrique comme une tentative/tentation de réponse formaliste à la nauséeuse matière postcoloniale.

Les nombreux problèmes posés par l'adaptation de textes littéraires au cinéma sont d'autant mieux connus que les classiques romanesques sont devenus des sources d'inspiration commodes pour des producteurs en mal de scenarii innovants. Et l'adaptation cinématographique de In Custody constitue à ce titre un cas d'école pour ce qui est de la divergence esthétique manifeste entre la romancière et le réalisateur. Ainsi, le chapitre trois du roman commence par une description d'un bazar de Delhi, où Deven pénètre pour la première fois, à la recherche du lieu où réside le vieux poète qu'il doit interviewer : «If it had not been for the colour and the noise, Chandni Chowk might have been a bazaar encountered in a nightmare " (IC 31). Ici, la couleur semble devoir participer à l'ancrage de la description dans un "réel» rendu positif par opposition à l'effet cauchemardesque du dédale des ruelles, de la chaleur et de la foule. Pourtant cette réalité a la couleur «rouge et vert-poison» des sorbets que le protagoniste refuse avec mépris, et les teintes criardes («lurid») des saris de nylon japonais qui lui font songer à des prostituées entreprenantes aux vêtements voyants ("gaudy »). On est bien loin, dans cette description, du décor recherché par Merchant qui a choisi de tourner le film non pas à Delhi, mais à Bhopal : «Delhi is too crowded. We wanted space and serenity and visual beauty (Meer). Cette priorité accordée à l'esthétique du cadre au détriment de la fidélité à l'imaginaire source apparaît tout aussi clairement dans la " colorisation » étonnante d'une brève scène du roman où il est suggéré que les deux femmes de Nur, enveloppées, pour l'une, d'un manteau brun et pour l'autre, d'un costume noir et argenté, vont en venir aux mains (93). Dans le film par contre, c'est un corps à corps somptueux qui est présenté au spectateur, l'une des femmes étant vêtue de bleu roi et l'autre de rouge carmin.

4 Le regret exprimé par Desai que son roman n'ait pas plutôt été tourné en noir et blanc prend donc avant tout sens par rapport à une divergence «éthique » entre un cinéma commercial, grand public (bien qu' « indépendants", les films produits par la maison Merchant and Ivory ont pour marque de fabrique des sets opulents et le recours fréquent à des stars), et un cinéma " engagé » le cinéma néoréaliste dont Satyajit Ray reste bien sûr la référence indienne la plus célèbre en Occident. Il s'agit donc de 
déterminer de quelle manière l'approche romanesque de Desai peut s'apparenter métaphoriquement au choix du noir et blanc du Nouveau Réalisme.

Contre ceux qui lui prêtent une vision tragique ou nostalgique, Desai se réclame plutôt d'un réalisme exigeant, sans concession : "I would prefer to think that the books face the truth and don't create illusions » (Costa).

6 L'objet de sa critique "réaliste » dans In Custody est d'abord le caractère stérile, mortifère de l'Inde contemporaine, incapable d'envisager l'avenir. Ceci est dû, pour une part, à sa soumission néocoloniale aux modèles américains et japonais de progrès technologique et de prospérité consumériste. Le roman montre comment, d'autre part, les crispations identitaires héritées de la Partition entraînent une polarisation communautariste conduisant à la confiscation revancharde par la minorité musulmane de la tradition poétique ourdoue, parallèlement à l'absence de tout soutien culturel, de la part de l'Establishment hindouiste, voire à une volonté politique active d'oblitérer le passé musulman. Dans son roman, Anita Desai insiste sur l'absence d'unité nationale indienne autour d'une histoire ou d'un territoire commun, et sur l'éclatement des références identitaires - qu'elles soient culturelles (décentrées vers le Pakistan ou le Moyen-Orient pour les Musulmans, du fait de la Partition ; ou encore vers l'Occident, du fait de l'acculturation coloniale) ou économiques, l'Amérique constituant un pôle d'attraction mirifique. À Mirpore, ville imaginaire emblématique des villes secondaires indiennes, cette absence de cohésion nationale et de valorisation d'une histoire commune se manifeste par un désintérêt institutionnel et populaire pour les monuments historiques et la tradition culturelle savante. Mais elle se manifeste également par l'absence de toute vision politique crédible et d'activité économique constructive. Ceci fait écho en outre à une sorte de fatalité du lieu, la sécheresse y étant chronique. La médiocrité de la petite ville provinciale n'est pas seulement subie, elle est assumée, et même recherchée : «Mirpore spared no effort to give an impression of total aridity » (IC 14). Cette médiocrité est donc rendue constamment visible et tangible par la poussière omniprésente qui homogénéise tout sous un voile de grisaille indifférenciée.

7 Par-delà le parallèle métaphorique entre l'éthique romanesque d'Anita Desai et l'esthétique noir et blanc du Nouveau Réalisme, se pose maintenant la question du traitement spécifique de la couleur dans le roman, et notamment pour «rendre » la médiocrité de cette ville, qui ne correspond à aucun lieu réel, mais dans laquelle il a été dit que l'on pouvait y reconnaître toutes les petites villes indiennes de province.

$\mathrm{Du}$ fait, comme nous l'avons vu, de l'omniprésence de la poussière, le gris y domine, prenant même le pas sur le rouge du sang versé les années où les commémorations chiites du martyr d'Hussein coïncident avec la fête hindouiste de Holi : « Knives flashed, batons flailed and blood ran. For a while tension was high [...]. Then the dust of Mirpore rose and swirled and buried everything in sight again; the citizens of Mirpore returned to their daily struggle to breathe » (IC 15).

9 L'action du roman étant concentrée sur les mois de saison sèche, les images de stérilité, de dessèchement et de mort prévalent dans les descriptions de la plaine autour de Mirpore, telle que la découvre Deven lors de ses trajets en bus vers Delhi. Le gris-blanc de la poussière s'associe alors au jaune de la végétation desséchée et des carcasses d'animaux disséminées sous un "ciel vide » pour suggérer un désert matériel, mais bien sûr aussi symbolique. Même si ici Desai ne cite pas The Waste Land de T.S. Eliot, comme elle l'avait fait dans Clear Light of Day, son roman précédent, ses descriptions du 
désert existentiel dans lequel Deven évolue en rappellent le pessimisme moderniste, pris entre la faillite des repères traditionnels et une modernité déshumanisante. Cependant, malgré un dessèchement extrême sans cesse réaffirmé, la grisaille de Desai n'accède jamais entièrement à l'abstraction du désert métaphysique. En effet, le gris, dans le roman, conserve l'empâtement, le poisseux de la matière. Le passage suivant, par exemple, s'apparente plutôt, dans son approche de la "réalité », au « kitchen sink realism» britannique des années soixante. Ici aussi les illusions romantiques se dissolvent dans la quotidienneté sordide d'un provincialisme étouffant :

Dawn was breaking at the end of the road as Deven rattled homewards in the bus. At least, that was how the poets described it, he thought bitterly as he looked through the dust filming the windowpane at the sooty darkness lightening to grey as if the soot and smuts were being dissolved in dishwater. [...] Dawn and poetry, he thought as he spat out a shred of tobacco from his mouth that felt painfully unclean at this hour of morning, all that was simply not real, not true. [...] Any reality was preferable, he told himself, even if it was the smeared window of a country bus bumping along the rutted road homewards. (62)

C'est avec une certaine complaisance, que la description s'appesantit ici sur la saleté, et ailleurs sur la décrépitude, voire le pourrissement, comme dans cet autre passage, qui se trouve à quelques pages du précédent :

The tedium [of his wife's sulking] settled upon him like a grey, crumbling mildew. He felt aged and mouldy. He was sure his teeth had loosened in the night, that his hair would come out in handfuls if he tugged it. That was what she might well do, he feared, to teach him not to venture out of the familiar, safe dustbin of their world [...]. Now he would sink back on to the dustheap like a crust thrown away, and moulder. (66)

La métaphore initiale de la moisissure qui illustre l'ennui, la déception et la résignation du personnage, perd de sa transparence conventionnelle et prolifère jusqu'à faire basculer le réalisme neutre dans un excès expressionniste qui rappelle plutôt Beckett. Bien sûr, il peut sembler paradoxal de parler d'expressionnisme du gris, si on le considère comme une non-couleur, assimilable à la simple absence de couleur " colorée», ainsi que de tout effet de contraste. La question se pose alors du rôle et du sens de la couleur résiduelle dans le roman, dans la mesure où elle est appelée à entrer en résonance ou en contraste avec la dominante idéologique grise.

Étant donné le contexte caniculaire, la principale couleur qui prolonge l'imaginaire de la médiocrité et de l'échec est naturellement le jaune : le jaune de la poussière, du ciel surchauffé, de la végétation desséchée $(15,20,21$, par exemple), des murs décrépits (17, 71), mais aussi le jaune des bonbons poisseux qui sont l'une des rares productions de Mirpore (16), des dents, des visages (142) ou des yeux bilieux (199), de la barbe de Nur, comparée à du vieux rembourrage de coussin (81), de la flaque de vomi (59) que Deven doit éponger avec des feuilles de papier qui sont peut-être des feuillets de poésie inédite. Les enveloppes de télégramme (154) et les cartes postales (139) le rappelant à Delhi pour d'autres humiliations sont également jaunes, ainsi que la facture graisseuse (205) pour la location de la chambre où pendant des semaines Deven a essayé en vain de faire un enregistrement audio de Nur. La couleur or est mentionnée une fois en référence au soleil qui jadis, dans un ciel non pollué, brillait comme un bijou princier (168), mais les autres occurrences l'associent aux dents couronnées, aux boutons de manchette d'un videur de maison close (161), ou aux broderies sur des saris racoleurs. Quant à la couleur safran des œillets d'Inde, c'est un memento mori : ils se fanent dans le bouquet compact préparé pour la réception organisée après le conseil d'administration annuel de l'université de Deven (100) et sont directement associés au deuil lorsqu'ils se 
mêlent à la cendre dont s'est barbouillé un sadhu (26), ou ornent le cercueil de Nur à la fin du roman (225).

Le rouge n'est pas très présent dans le roman, même si la plupart des monuments du vieux Delhi sont construits avec du grès rouge. Comme nous l'avons vu plus haut, la vision dramatique du sang répandu lors d'affrontements entre Hindous et Musulmans n'est que suggérée et vite escamotée par le gris-poussière. L'épisode du chien heurté par le bus ne donne pas non plus lieu à l'irruption du rouge: les passagers sont asphyxiés par le nuage de poussière jaune levé par l'écart qu'a fait le bus pour tenter d'éviter le chien, et une volée de corbeaux dérobe la victime à la vue de Deven, qui serait prêt à interpréter cet incident comme un signe avant-coureur de tragédie. Pudique face à la vraie tragédie, le regard se fait beaucoup plus insistant devant la violence sordide de l'avidité ordinaire. Ainsi les bouches sont volontiers dévorantes dans le roman, et les dents soulignées du rouge du bétel. Murad, dont l'haleine est infecte, ne cesse d'en mâcher, et la prise de bétel joue également un rôle important dans la description du récital que donne Imtiaz Begum, la seconde épouse de Nur, agressivement fardée, comme pour montrer à tous qu'elle a définitivement supplanté son mentor : "Someone brought a silver box of betel nuts and leaves-the smile Imtiaz Begum gave was as sudden and swift as if scissors had cut it through her face, snipsnap, and the teeth were stained red besides " (83). Très clairement, ce n'est pas elle, avec son visage et ses lèvres fardées, et ses ongles de main et de pieds peints, qui est la victime, mais bien celle qui brandit symboliquement les ciseaux castrateurs. Dans le domaine $\mathrm{du}$ fard, le rouge fonctionne comme le noir: celui des mèches teintes (" horrendously dyed black hair » 125), qui s'enroulent à terre comme des serpents, ou celui du khol qui est censé embellir les yeux, souligne plutôt la violence et la laideur sous-jacentes : "The tall woman with the pock-marked face, holding a pink veil across it, glared through two huge rims of kohl » (188).

On pourrait s'attendre à ce que les touches de vert et de bleu viennent apporter un peu de réconfort dans un univers caractérisé par le manque d'eau, de vitalité, de fraîcheur. Le vert joue effectivement très fugacement ce rôle dans une scène de complicité inattendue entre Deven et son fils. Ayant décidé de se réconcilier avec la médiocrité de sa vie professionnelle et familiale à Mirpore, Deven vit avec le jeune Manu un moment de joie inespérée, emblématisée par une plume verte de perroquet: "One brilliant feather of spring green fluttered down through the air and fell at their feet in the grey clay. Deven bent to pick it up and presented it to his son who stuck it behind his ear [...]. Yes, it was the climax of that brief halcyon passage. It was as if the evening star shone through at that moment, casting a small pale illumination upon Deven's flattened grey world » (74). Mais c'est la seule fois que le vert apparaît dans un contexte positif. Ailleurs dans le roman, le vert est celui des réservoirs d'eau stagnante de Mirpore (14), de la végétation poussée aux engrais du collège d'agronomie (17), de la chemise verte en nylon (18), présent des beaux-parents que Deven méprise mais dont il doit accepter les largesses parce qu'il est plus pauvre qu'eux. Desai a également choisi le vert pour les ongles d'un laitier dans le bus (63) et le bandeau sur l'œil d'un autre passager (21), ainsi que pour les boissons douteuses vendues dans la rue, les enseignes au néon (53), les murs sinistres de l'hôpital en face de chez Nur $(32,33,210)$ et, parallèlement, le carrelage vert foncé des murs à l'intérieur de la maison du poète (36, 118). On peut encore mentionner le mouchoir vert sale (30) derrière lequel Murad, qui se prétend son ami, semble encore une fois se payer la tête de Deven, qui en devient... 
gris d'appréhension. Le vert est souvent agressif, corrosif ou chimique (" acid green », " poison-green ", « chemically lush», " fertilizer-fed green »). Contrairement au film, qui rappelle que le vert est la couleur du paradis pour l'Islam, en plaçant un dais vert sur le cercueil de Nur, le roman montre comment Nur et le groupe de ses admirateurs musulmans associent avec mépris le vert à la culture hindoue, réduite à une alimentation végétarienne et au culte des vaches sacrées ( Oh Hindi is a field of greens ", 54).

Lorsqu'il est associé au bleu, comme dans le rideau de perles de verre qui se referme sur l'échec de Deven à la fin des séances d'enregistrement, le vert signifie davantage la désillusion: "He let go of the strings of [blue and green] beads that tinkled glassily against each other, again reminding him of something: Sarla's glass bangles clinking as she worked? Home, Mirpore, quotidian life drawing close, closing in? » (187). « Again » renvoie au fait que le quotidien morne de Mirpore vient également de lui être remis en mémoire par le cri plaintif du vendeur d'eau, évoqué cinquante pages plus tôt (139). Ici, le vert ramène donc explicitement au gris-poussière dominant. Dans ce roman du dessèchement, la couleur bleue n'apparaît que très rarement, et s'avère illusoire ou décevante: la fontaine asséchée de Mirpore est peinte en bleu (15), ainsi que le distributeur d'eau installé à l'université au cours des préparatifs du conseil d'administration, mais sur lequel des graffitis apparaissent immédiatement et qui, parce qu'il fuit, est condamné à rouiller au milieu d'une flaque de boue (97). Lorsqu'il est support d'écriture ourdoue, le bleu renvoie à une aspiration à un idéal. Ainsi, les quelques lignes griffonnées par Siddiqui pour annoncer à Deven que l'université va financer l'achat d'un magnétophone semblent promettre une libération - qui s'avérera bien sûr illusoire : "Seeing that line waver and break up and come together again upon the sheet of blue paper, Deven felt as if he were seeing all the straight lines and cramped alphabet of his small tight life wavering and dissolving and making way for a wave of freshness, motion, even kinesis » (110). De la même façon, le volume de poèmes que Deven imagine relié en bleu pâle avec un titre reproduisant la calligraphie de Nur ne sera jamais publié, car les poèmes qui lui parviendront à la fin du roman, calligraphiés sur papier bleu, ne seront que ceux d'Imtiaz Begum, et finiront donc au rebut : "Sarla, coming in with her duster, looked at him tearing up a bundle of blue sheets of paper into strips, and cried, "You're dropping rubbish all over the floor I have just swept!" » (217).

14 Après ce tour d'horizon non exhaustif, il est possible de conclure que le traitement de la couleur dans In Custody est fort différent de celui que l'on trouve non seulement dans l'adaptation de Merchant, mais également dans d'autres romans de Desai. Dans Bye, Bye Blackbird, par exemple, la couleur se fait sensuelle, étourdissante, alors qu'ici elle fonctionne essentiellement dans le champ sémantique de l'horreur nauséeuse. Ainsi, les couleurs qui illuminent un ciel vespéral bien peu serein forment un tableau qui est loin de correspondre à la séduction exotique d'une Inde en Technicolor: "The white-hot sky became blotched with the city's soot and faded to grey and then to mauve and finally to an uneasy, disturbed darkness. It could not become wholly dark because then the shops and cinema houses and restaurants and streets of the bazaar below lit up for the night and the sky was tinged sporadically red and orange and yellow and violet, like an old hag at a fair crazily dancing a dance of seven veils » (46).

La couleur est donc particulièrement horrible parce qu'elle orne monstrueusement une réalité mortifère. Mais d'où peut venir cette logique de mort qui affecte les couleurs? 
Le dégoût et le mépris qui caractérisent Deven dans ses rapports aux objets et personnes qui l'entourent, sa crainte constante de la contamination ou de la souillure physique ou morale, pourraient orienter l'interprétation vers une explication culturelle liée aux interdits religieux, tant hindouistes que musulmans. Comme l'écrit Luc de Heusch dans la préface d'un ouvrage précisément sur le concept de souillure: «Le drame de la culture indienne est [...] qu'elle est fondée sur l'extension métaphorique de la nausée. [...] L'Inde nous offre ainsi l'étrange et dramatique spectacle d'un univers où le délire métaphorique de la souillure règne en maître sur la pensée, investit toutes les prohibitions, toutes les classifications. [...] Tout homme risque sans cesse d'être entraîné dans l'abominable et de déchoir » (Douglas 18-19).

Cependant, il existe dans In Custody un net parti-pris en faveur du décloisonnement culturel, qui semble aussi aller, au delà-même du syncrétisme, à l'encontre d'une interprétation strictement religieuse du monde. En effet, Deven Sharma, qui, d'après son nom, serait d'origine brahmine, ne pratique aucun rituel (contrairement à Sarla qui fait des offrandes à Lakshmi, la déesse de la chance). Il accepte même de consommer des plats comportant de la viande. Enfin, il a l'ourdou pour langue maternelle et la poésie ourdoue pour seule passion, bien que dans son université, il soit affecté au département de littérature hindoue. Le fanatisme religieux est d'ailleurs explicitement dénoncé dans le roman, comme dans le passage où le visage d'une voisine que Deven déteste particulièrement est décrit comme étant à la fois "moralisateur et martial comme une hachette entre les mains d'un fanatique » (65).

Il semble donc possible de détacher In Custody de l'imaginaire de la souillure fatidique, spécifiquement religieux. À la place, le parallèle que le roman établit entre l'obsession du sale (poussière, pourriture ou déjection) et la désespérance existentielle se déploie dans un contexte de découragement idéologique face à une impossible dynamique de progrès que métaphorise l'impossibilité d'histoire. Une longue description de Mirpore au début de In Custody fait ainsi apparaître une humanité matérielle et sociale qui s'obstine dans une régénérescence à l'identique, synonyme, paradoxalement, de mort :

Those shacks of tin and rags, however precarious and impermanent they looked, must have existed always, repetitively and in succeeding generations, but never fundamentally changing and in that sense enduring. The roads that ran between their crooked rows had been periodically laid with tar but the dust beneath was always present, always perceptible. In fact, it managed to escape from under the asphalt and to rise and spread through the town, summer and winter, a constant presence, thick enough to be seen and felt. (Desai 12)

Cependant, In Custody n'a pas le pessimisme radical d'un univers qui serait à la fois privé de dynamique et de transcendance. C'est en vérité par l'éclosion de la seconde que la première, finalement, retrouve sens et figure, quoique de façon inattendue.

En effet, la fin du roman érige Deven en une sorte de figure christique de l'amitié, pathétique et résignée, faisant de ce point de vue écho aux dernières pages de Clear Light of Day, où le personnage central accepte de sacrifier ses ambitions personnelles sur l'autel de l'amour de l'autre, troquant un destin héroïque contre une routine ancillaire et victimaire. Conformément à l'imaginaire christique, le sacrifice est héroïsé par la violence subie. Or, dans cette dernière scène de In Custody, le gris est à nouveau présent, mais seulement comme une transition entre le noir du désespoir et la blancheur du renouveau et de la joie : "The sky was filling with a grey light that was dissolving the dense blackness of night. It glistened upon a field of white pampas grass 
which waved in a sudden breeze that had sprung up, laughing, waving and rustling through the grasses with a live, rippling sound » (224).

Toutefois, le caractère quelque peu cliché de la référence chrétienne au souffle divin, aux calamités s'abattant du ciel telles des épées pour faucher Deven, et à la branche d'épines qui entrave un instant son retour éperdu vers ses responsabilités, ne permet pas de trouver cette fin entièrement convaincante, ne serait-ce que parce qu'elle semblerait cautionner le retour du religieux, tout investi d'une dynamique de la Passion qu'il soit. Cette scène renvoie par contraste à une autre où le noir et blanc sont également présents, mais cette fois-ci dans un contexte où l'intense émotion du personnage semble être entièrement d'ordre esthétique, sans aucune connotation religieuse (au sens de doctrinale ou textuelle) malgré l'objet qui la suscite. Il s'agit d'un passage qui se trouve à une dizaine de pages de la fin du roman, alors que Deven est ruiné, déshonoré, trahi par ceux qu'il prenait pour ses amis alors qu'il croule sous diverses demandes de faveurs qu'elles soient ou non pécuniaires. Errant entre le bazaar de Chandni Chowk, le Fort Rouge, et la Mosquée du Vendredi, Deven comprend enfin la nature de l'indicible au cœur de la poésie du vieux Nur Shahjehanabadi, dont le nom signifie précisément «la cité du Shah Jahân ${ }^{1}$ ", c'est-à-dire le vieux Delhi dans lequel le personnage déambule dans cette scène. Dans un parc où tout est "calmement géométrique ", Deven accède à un moment de paix grâce au spectacle de l'entrée principale de la grande mosquée, qui domine les toits du vieux Delhi :

The sun was behind it, in a great brassy conflagration, dazzling his eyes, but its forms and lines stood out against the heat and light clearly. The white and black marble facing of the eastern doorway made a graceful calligraphic pattern. The enormous arched doorway soared upwards to the dome which rose like a vast bubble that the flat earth had sent out into the dusty yellow-grey sky, a silent exhalation of stone. It was absolutely still, very serene. It was in fact the silent answer to his questioning. (211)

L'éloquence formelle de la combinaison de l'abstraction géométrique et de la calligraphie donne ici au contraste entre le noir et le blanc un sens très différent de celui que l'on trouve dans la scène précédemment évoquée. Ici, le contraste ne relève plus d'un symbolisme manichéen. La structure de la phrase souligne que c'est de l'association du blanc et du noir que naît une gracieuse calligraphie. Celle-ci étant par ailleurs dépouillée de son caractère de texte et décrite seulement comme un "motif", elle ne se prête pas au décodage religieux au sens strict. Ce n'est pas qu'elle soit d'une religiosité rendue illisible par la saleté et le vandalisme oisif, comme dans la mosquée du début du roman qui, bien que méconnaissable, est « utilisée » cinq fois par jour pour la prière (13). C'est que le plaisir esthétique et a-doctrinal procuré par la calligraphie est présenté comme signalant lui-même le seuil d'un passage vers un absolu transcendantal. Le motif calligraphique orne avec légèreté et grâce le gigantisme architectural pour accompagner une révélation qui ouvre à une méditation sur la forme pure : «Gradually the sky disappeared, the sun and the light and the glare, and the shape became clearer and sharper till it was all there was-cool, high-minded and remote " (211). Cette contemplation d'un absolu, formel et non plus littéral, permet à Deven de s'abstraire pour la première fois du monde qui l'entoure. Lorsque la profusion criarde et colorée de celui-ci s'impose à nouveau, Deven comprend en quoi la perfection de la forme pure, non-figurative, non-expressive est nécessaire :

If art, if poetry, could be made to submit their answers, not merely contain them within perfect, unblemished shapes but to release them and make them available, then-he thought, then- 
But then the bubble would be breached and burst, and it would no longer be perfect. And if it were not perfect, and constant, then it would all have been for nothing, it would be nothing. (212)

Renonçant à attendre de l'art une "réponse " à son angoisse existentielle, Deven s'abîme dans la contemplation de la forme parfaite, qui ne manifeste l'idéal qu'elle « contient » qu'en le rendant inaccessible.

Entre calligraphie et géométrie, ce passage renvoie chez Desai, elle-même, à une esthétique de l'épure. En effet, elle qui, même dans ses romans, a toujours privilégié la concision, s'est dit de plus en plus attirée par la densité de la forme poétique : «I would like to achieve that gravity, compression, the intensity of their language " (Costa). Cependant, l'exigence réaliste, telle que Desai la conçoit et l'assume dans son travail romanesque, ne lui permettait sans doute pas de conclure sur une vision d'harmonie abstraite ce roman, qui est peut-être celui qui est le plus engagé dans la mesure où il dénonce explicitement les politiques culturelles de l'Inde d'après la Partition ${ }^{2}$. Néanmoins, c'est cette forme parfaite, à la fois circonscrite et indescriptible, qui donne sens aux péripéties les plus désastreuses, passées et à venir, de la quête de Deven, comme le rappelle la formule citée plus haut, à travers un jeu d'une sobriété magnifique sur la pluri-référentialité du pronom indéfini : « if it were not perfect [...], then it would all have been for nothing, it would be nothing ». Quels que soient le dégoût et le découragement que suscite une réalité matérielle et humaine sordide, l'engagement est ici rendu d'autant plus nécessaire que la culture ourdoue, emblématisée à Delhi par le règne du Shah Jahân, est réellement en danger. C'est ce que rappelle, au onzième et dernier chapitre, la destruction de la haveli de Siddiqui, dernier descendant d'un nabab, ainsi que la mort des pigeons de Nur, symboles de son inspiration poétique, et qui sont étouffés par une « moisissure grise » qui leur ferme les yeux, leur clôt le bec et finit par les étouffer (221).

21 Bien que mondialement reconnue, Desai n'a jamais été récompensée par les prix les plus prestigieux, même si trois de ses romans (dont In Custody) ont été présélectionnés pour le Man Booker Prize. Le format court, l'univers assez étouffant et la vision pessimiste de ses romans et de ses nouvelles sont à contre-courant de l'esthétique baroque, gargantuesque, touffue et bariolée d'un Salman Rushdie, qui correspond peutêtre davantage à la vision du monde indien définie par les attentes d'un public occidental. Ainsi, les stéréotypes orientalistes transparaissent encore dans le jugement quelque peu condescendant, rapporté ici, de l'adaptation cinématographique de In Custody: "Merchant's film has a mood, a texture, and composition that is disarmingly vital and colourful, blending the many sights, sounds, and traditions of India into one great untidy whole » (Spring). Le critique déploie le paradigme de l'irrationnel attrait de la matière orientale en évoquant la séduction « désarmante ", vibrante et colorée de la profusion échevelée. On peinera peut-être à y reconnaître une description convaincante du film, mais cet imaginaire est de toute façon complètement étranger à l'œuvre de Desai, qui, si l'on se risque à reprendre des catégories commodes, relève davantage d'une économie plus «classique» que «baroque». Loin de l'exotisme séduisant auquel Merchant se laisse ponctuellement aller dans l'adaptation du roman, In Custody propose, au contraire, un traitement « politique » de la couleur, dans le cadre d'une réflexion éthique et esthétique sur les « valeurs » du noir et blanc, qu'elles soient celles du néoréalisme engagé ou celles de l'abstraction formelle. 


\section{BIBLIOGRAPHIE}

Costa, Magda. "Interview with Anita Desai”, 30 Jan 2001 : www.sawnet.org/books/writing/desaiinterview.html, consulté le 26/01/2015.

De Heusch, Luc. Préface de Mary Douglas. De la Souillure. Paris : François Maspero, 1981.

Desai, Anita. Fire on the Mountain (1977). London: Vintage, Random House, 2001.

Desai, Anita. Clear Light of Day (1980). London: Vintage, Random House, 2007.

Desai, Anita. In Custody (1984). London: Vintage, Random House, 1999.

Dvorak, Marta. The Faces of Carnival in Anita Desai's In Custody. Paris : PUF, 2008.

Ganapathy-Doré, Geetha. “The Lyrical and the Imagistic in Anita Desai's In Custody”. GanapathyDoré (ed.). Revisiting Anita Desai’s In Custody for the Agrégation. Sari (2009): 167-188.

Khan, Nyla Ali. "Places and Politics of Identity in Anita Desai's In Custody". The Atlantic Review 5.1-2 (Jan-March \& Apr-June 2004): 128-145.

Meer, Amena. “Interview of Ismail Merchant”. Interview (New York), April 1994.

Robinson, Andrew. “Satyajit Ray : A Moral Attitude”. Sight and Sound 23.9 (Sept 2013): 42-45.

Spring, Justin. “One for all”. Artforum 01/02/2006.

\section{NOTES}

1. 局E Empereur Moghol dont le règne s'étend de 1627 à 1658 , et dont le nom reste associé à la construction, outre de la Jama Masjid de Delhi (la plus grande mosquée de l'Inde), du Taj Mahal à Âgrâ, du Fort, des Jardins de Shalimar et de la mosquée de Wazir-Khan à Lahore.

2. 㡠Cette tension entre l'épure et le foisonnement sordide est souvent présente dans l'œuvre de Desai, mais dans un roman comme Fire on the Mountain, la première est plus explicitement dénoncée comme un mensonge lâche et coupable. Nanda Kaul a trouvé refuge dans la solitude et le dépouillement d'un bungalow dans l'Himalaya, pour oublier son incapacité à aimer et à être aimée. Mais l'irruption d'abord de son arrière-petite-fille (une enfant asociale que ne rebute ni la laideur de l'usine pharmaceutique voisine ni la violence du monde naturel), puis de son amie d'enfance (une vieillarde-enfant grotesque qui malgré son propre dénuement, se dévoue pour tenter d'améliorer les conditions de vie misérables des femmes du village voisin), font exploser la «bulle » élégante dans laquelle s'était refugiée Nanda.

\section{RÉSUMÉS}

Anita Desai, romancière d'une Inde «sobre » est dans la tradition du réalisme psychologique à tendance mystique qu'on opposera à la tradition baroque, "orientaliste", pratiquant un symbolisme descriptif volontiers bariolé. Chez Desai, nulle célébration de la beauté du monde, 
pas d'univers chaotique en pleine construction, mais un ordre ancien moribond. Quel peut y être le statut de la couleur, qui ne dit ni la vitalité euphorisante, ni la splendeur décadente du vestige, chère à la tradition symboliste? Interrogée sur l'adaptation cinématographique de son roman In Custody, Desai affirme avoir été gênée de voir l'univers romanesque qu'elle avait imaginé si terne tourné « en Technicolor ", ajoutant qu'elle aurait préféré le voir transposé en noir en blanc. Le roman convoque pourtant, certes sur fond de grisaille, des couleurs souvent violentes dont la discordance contribue indéniablement à l'efficacité symbolique et visuelle des tableaux. Cependant, si le recours à la couleur relève bien du métier de l'écrivain « réaliste ", c'est autour de l'opposition entre le noir et le blanc que se construit la quête du sens et de la « forme poétique parfaite ». On s'interrogera sur le sens « romantique» du personnage central, dont le regard exalte la non-couleur du monde, qui elle-même valorise la concentration intérieure et l'aspiration à l'ailleurs. Le gris accède au statut de couleur mystique et le noir et blanc deviennent les couleurs d'une écriture du monde, d'une calligraphie, par opposition à la coloration criarde, synonyme d'indigence matérielle et spirituelle, sans profondeur ni poésie.

In this novel, Anita Desai offers a stark depiction of Post-Partition India, and clearly places herself within the tradition of psychological realism that can be contrasted with the baroque, richly coloured orientalist tradition. Nowhere in In Custody does Desai indulge in a celebration of the beauty of an exotic, chaotic but vibrant world. Rather, the India she unconventionally depicts is a dreary existential desert. This paper will explore what role colour is given to play when it may neither stand for oriental vitalism nor for the decadent but majestic ruins of a glorious past so dear to the symbolist tradition. When she was asked to give her opinion about Ismael Merchant's film adaptation of her novel, Desai answered she had been shocked to see that the world of the novel she had visualized all in grey had been shot in "gorgeous Technicolor", adding that she would have preferred the black and white of New Realism. Nevertheless, in spite of the prevailing greyness of the novel, colours do appear, and their "lurid" and "gaudy" presence contributes to the symbolic and visual efficiency of Desai's portrayal of late 20th century India. However, beyond the postcolonial writer's "realist" use of colour, it does seem as if the quest for meaning and the "pure" poetic form is structured around the contrast between black and white. In the eyes of the idealistic main character who aspires to poetic transcendence, the aesthetic experience of calligraphy takes on a mystical meaning, offering relief from the garish paucity of spirituality and tradition of modern India.

INDEX

Keywords : new realism, black and white, colour, exoticism oeuvrecitee In Custody

Mots-clés : néoréalisme, noir et blanc, couleur, exotisme

\section{AUTEURS}

\section{ANNE LE GUELLEC-MINEL}

Anne Le Guellec-Minel est Maître de Conférences à l'Université de Brest. Après une thèse sur l'épique romanesque dans l'œuvre de Patrick White, elle s'est spécialisée en littérature australienne, publiant des articles sur Patrick White, David Foster, Peter Carey, David Malouf et Richard Flanagan, notamment. Elle s'est également intéressée à la littérature aborigène, avec Kim Scott, Alexis Wright et Bruce Pascoe. Au-delà de la littérature australienne, elle travaille sur les littératures du Commonwealth dans une approche postcoloniale. Elle a rassemblé un recueil 
d'articles intitulé Voix défendues pour les Cahiers du CEIMA (2012) et travaille sur un autre recueil sur les rapports de force entre la mémoire et l'Histoire. 